\title{
Metas de realização, autorregulação da aprendizagem e autopercepção de desempenho em universitários
}

\author{
Simone Nenê Portela Dalbosco \\ Adriana Satico Ferraz \\ Acácia Aparecida Angeli dos Santos \\ Universidade São Francisco, Campinas-SP, Brasil
}

\section{Resumo}

A presente pesquisa aferiu as correlações e o nível preditivo entre as metas de realização e a autorregulação da aprendizagem e, também, verificou as suas diferenças em razão da autopercepção de desempenho dos estudantes. Participaram do estudo 404 universitários ingressantes $\left(M_{\text {idade }}=19,62 ; D P=4,870\right)$. Foram aplicados coletivamente um Questionário Sociodemográfico, Inventário de Processos de Autorregulação da Aprendizagem-IPAA e Escala de Avaliação da Motivação para a Aprendizagem-EMAPRE-U. Identificou-se correlação entre o IPAA e a meta aprender $(r=0,24)$. As metas aprender e performance aproximação se mostraram preditoras dos escores do IPAA. Os estudantes com alta percepção de desempenho acadêmico obtiveram maior média na meta aprender e para aqueles com baixa autopercepção a maior média foi na meta performance evitação.

Palavras-chave: Motivação, aprendizagem, rendimento escolar

Abstract: Achievement goals, self-regulation of learning and self-perception of performance in undergraduates The present study assessed the correlations and the predictive level between achievement goals and self-regulated learning, and verified the differences in self-perception of undergraduates' academic performance in both constructs. Participated in this study 404 undergraduates $\left(M_{\text {age }}=19.62, S D=4.870\right)$. The instruments used in the collective data collection were the Sociodemografic Questionnaire, the Inventory of Learning Self-Regulation Processes-IPAA and the Motivation for Learning Assessment Scale-EMAPRE-U. A correlation was identified between the IPAA and the learning goal $(r=0.24)$. The learning goal and performance-approach goal proved to be predictors of the IPAA. Undergraduates with high perceptions of academic achievement scored higher on the learning goal, while those with low self-perception scored higher on performance-avoidance goal.

Keywords: Motivation, learning, school performance

Resumen: Metas de realización, autorregulación del aprendizaje y autopercepción de desempeño en universitarios La presente investigación evaluó las correlaciones y el nivel predictivo entre las metas de realización y autorregulación del aprendizaje; verificó además sus diferencias con relación a la autopercepción del desempeño de los estudiantes. En el estudio participaron 404 universitarios de reciente ingreso $\left(M_{\text {edad }}=19,62 ; D P=4,870\right)$. Se aplicaron colectivamente un Cuestionario Sociodemográfico, Inventario de Procesos de Autorregulación del Aprendizaje-IPAA y Escala de Evaluación de la Motivación para el Aprendizaje-EMAPRE-U. Se identificó correlación entre el IPAA y la meta aprender $(r=0,24)$. Las metas aprender y la meta performance aproximación se mostraron predictores de las puntuaciones del IPAA. Los estudiantes con alta percepción de desempeño académico obtuvieron mayor promedio en la meta aprender y para aquellos con baja autopercepción la mayor media fue en la meta performance evitación. Palabras clave: Motivación, aprendizaje, desempeño escolar

${ }^{1}$ Endereço para correspondência: Avenida Major João Schell, 680, B. Fátima, 99020-020, Passo Fundo, RS. E-mail: snpdalbosco@gmail.com 
As instituições de Ensino Superior e, principalmente, o professor são constantemente desafiados a identificar e lidar com as dificuldades dos estudantes, em especial daqueles que acabaram de ingressar. Dentre os aspectos que podem dificultar a sua adaptação a este nível de ensino se destacam as exigências do curso, que requerem do estudante maior proatividade nas aulas, organização para estudar e realizar as atividades, bem como o gerenciamento do tempo para conciliar os estudos com a vida fora do contexto acadêmico. As disciplinas também exigem o desenvolvimento de competências novas para muitos dos estudantes, como é o caso das habilidades de argumentação e de investigação. Portanto, é fundamental que o estudante esteja motivado para se envolver ativamente com a sua própria aprendizagem, principalmente no que se refere à regulação do esforço empreendido nas tarefas $\mathrm{e}$ a persistência diante das adversidades (Mega, Ranconi, \& De Beni, 2014; Santos \& Mognon, 2016).

Buscando compreender o funcionamento de alguns dos aspectos subjacentes ao processo de ensino e aprendizagem, este estudo centralizou-se na investigação da motivação do aluno para aprender (metas de realização) e da autorregulação da aprendizagem. Torna-se relevante a investigação de ambos os construtos, uma vez que estão presentes na rotina dos estudantes, permeando o modo com eles encaram as atividades acadêmicas, estabelecem os objetivos de aprendizagem e se organizam para realiza-las (Bzuneck \& Boruchovitch, 2016; Mega et al., 2014; Zimmerman, 2013). Conjectura-se que este estudo poderá auxiliar os profissionais envolvidos no Ensino Superior a compreenderem a importância da estimulação e manutenção da motivação do estudante, fazendo com que se sintam mais capazes de promover a autorregulação da aprendizagem.

A motivação para aprender, sob a perspectiva das metas de realização, se constituem de crenças expressas pelos pensamentos, sentimentos e comportamentos do estudante em relação às diversas situações acadêmicas, como é o caso das atividades feitas em sala de aula, individualmente ou em grupo e a participação nas aulas (Bzuneck \& Boruchovitch, 2016; Daniels et al., 2013). As metas de realização são classificadas em consonância à percepção do estudante sobre o motivo que o leva a estudar, sendo possível observar, em uma mesma sala de aula, estudantes mais orientados para a meta aprender e/ou pelas metas performances aproximação e evitação (Bzuneck \& Boruchovitch, 2016; Pekrun, 2016).

O sucesso acadêmico para o estudante orientado majoritariamente pela meta aprender, configura-se pelo aumento de conhecimentos e de suas habilidades. Para atingir os seus objetivos de aprendizagem o estudante com esse perfil motivacional empreende esforços para realizar as atividades acadêmicas, e em situações de fracasso como, por exemplo, ao tirar uma nota baixa, consegue extrair das devolutivas feitas pelos professores elementos que o ajudem a melhorar, assim como busca aprender com os próprios erros (Bzuneck \& Boruchovitch, 2016; Grant $\&$ Dweck, 2003).

Por conseguinte, a orientação pela meta performance aproximação é identificada no estudante que percebe o sucesso ou insucesso acadêmico com base na comparação dos seus resultados com o de colegas de turma ou com base no reconhecimento dos professores. Por conta disso, esses estudantes têm a necessidade de tirar notas altas, manifestam grande satisfação em demonstrar publicamente que dominam determinado assunto e buscam liderar os grupos de estudos que participam (Bzuneck \& Boruchovitch, 2016; Bzuneck, Boruchovitch, Miranda, \& Almeida, 2014). Em virtude destas características, apesar de comumente apresentar notas altas, o aluno que possui predominância na meta performance aproximação pode apresentar estratégias cognitivas superficiais e baixa percepção de sucesso acadêmico, uma vez que a sua preocupação centraliza-se no desempenho e não na qualidade da aprendizagem em si (Bzuneck et al., 2014; Daniels et al., 2008).

A meta performance evitação, última meta explorada neste estudo, é marcada pela percepção de competência negativa, expressa pela sensação de inferioridade e incapacidade. $\mathrm{O}$ estudante que possui esse perfil motivacional é identificado por sua baixa persistência, já que não percebe, por exemplo, a possibilidade de regular os seus esforços de acordo com as demandas do contexto acadêmico. Essa meta também se apresenta como pouco funcional à saúde psicológica do estudante, já que pode ser uma fonte geradora de ansiedade, principalmente em situações avaliativas e de divulgação das notas das provas. Por conta destas características, a orientação pela meta performance evitação pode interferir negativamente na motivação do aluno para aprender, sendo associada à desistência (Anderman, Anderman, Yough, \& Gimbert, 2010; Bzuneck \& Boruchovitch, 2016; Daniels et al., 2013).

No tocante à investigação das metas de realização no Ensino Superior, o estudo desenvolvido por Daniels et al. (2013) identificou a associação entre as metas performances e o coeficiente de rendimento acadêmico dos alunos. Apesar de se tratar de realidades diferentes, esse resultado corrobora a posição de Bzuneck (2009), que já salientava que as metas performances tendem a favorecer o sistema de ensino baseado em notas. As metas performances também se associaram à sensação de vergonha 
em sala de aula e se mostraram divergentes em relação à autoeficácia acadêmica e social (Rosas, 2015). Estas metas também estiveram associadas às estratégias autoprejudiciais, que se referem às desculpas dadas pelo estudante após uma situação de fracasso, com a finalidade de proteção do impacto emocional negativo pelo ocorrido (Ferradás, Freire, Nuñez, Piñeiro, \& Rosário, 2016). Estas pesquisas também evidenciaram que a meta aprender auxilia os estudantes a lidarem e a se adaptarem ao ambiente acadêmico, à medida que se associa ao controle dos pensamentos e comportamentos na promoção do ajustamento ao ambiente, ao mesmo tempo que minimiza a ocorrência de manifestações de sentimento de vergonha em sala (Daniels et al., 2013; Rosas, 2015).

As metas de realização, além de estarem associadas ao modo como o estudante lida com a rotina acadêmica - estabelecimento de metas e objetivos, também fazem parte do processo da autorregulação da aprendizagem (ARA). Este processo tem por característica principal oferecer recursos para que o estudante consiga estabelecer e cumprir objetivos direcionados à rotina acadêmica, partindo do pressuposto de que o estudante pode ser um agente ativo da sua aprendizagem (Zimmerman, Schunk, \& DiBenedetto, 2015). A ARA é constituída por quatro aspectos principais, de ordem cognitiva, afetiva, comportamental e ambiental. $\mathrm{O}$ aspecto cognitivo se expressa, por exemplo, pela capacidade do aluno de antecipar as consequências de suas ações; o afetivo é representado pela motivação; o comportamental, ligado às ações do aluno; e o ambiental refere-se às relações estabelecidas pelo estudante com o meio (Schunk, Usher, \& Barry, 2013; Zimmerman et al., 2015). Segundo Mega et al. (2014) e Rosário, Fuentes, Beuchat e Ramaciotti (2016), estudantes autorreguladores são capazes de gerenciar o tempo, criar estratégias para estudar, executar as tarefas acadêmicas, individuais ou em grupo, se preparar para as avaliações e realizar uma autoavaliação sobre a própria aprendizagem e desempenho.

O processo da autorregulação da aprendizagem, segundo Zimmerman $(2000,2013)$ ocorre de forma cíclica e envolve três fases, denominadas de fase prévia, fase de realização e fase de autorreflexão. Durante a fase prévia o estudante analisa a tarefa. Esta etapa engloba as crenças motivacionais do estudante expressas pela autoeficácia, definida pelo quanto esse acredita ser capaz de realizar a atividade; a motivação intrínseca, associada ao comprometimento do aluno motivado a aprender sem a necessidade de cobranças e recompensas externas e as metas de aprendizagem, representada pelas metas de realização, construto motivacional e objeto de investigação do presente estudo (Zimmerman et al., 2015).
$\mathrm{Na}$ fase seguinte, de realização, ocorre a execução da tarefa e dois subprocessos acontecem concomitantemente, o autocontrole e a auto-observação. $\mathrm{O}$ autocontrole compreende as estratégias da tarefa, estratégias volitivas, autoinstruções, gerenciamento do tempo, características ambientais, comportamento de pedir ajuda, o interesse e a capacidade de prever as consequências. Por sua vez, a auto-observação envolve o monitoramento metacognitivo e a autorrecordação, em que o estudante mantém o foco no conteúdo e na forma que está aprendendo (Schunk et al., 2013; Zimmerman et al., 2015).

Na última fase, nomeada de autorreflexão, o estudante é levado a realizar o autojulgamento sobre as situações de sucesso e fracasso acadêmico vivenciados, denominado de atribuições de causalidade (Weiner, 2010). O julgamento do próprio desempenho leva a três tipos de autorreações: a autossatisfação, que aumenta a motivação para aprender; as inferências adaptativas, que em uma situação de fracasso leva o estudante a refletir sobre como reverter essa situação; e as inferências defensivas, em que o estudante se esforça para preservar sua autoimagem em situações de fracasso. Nesse último, o aluno pode, por exemplo, faltar nas aulas e passar a não cumprir os prazos estabelecidos (Zimmerman, 2011).

Em Wolters e Hussain (2015), alguns aspectos da ARA se mostraram favoráveis ao desempenho acadêmico de estudantes universitários, a saber, as suas estratégias cognitivas e metacognitivas, o gerenciamento do tempo e do ambiente e a motivação, enquanto que a procrastinação, caracterizada como uma estratégia autoprejudicial à aprendizagem se mostrou desfavorável ao desempenho acadêmico. As metas de realização estiveram associadas aos componentes da ARA, referentes à execução da tarefa, organização nos estudos, metacognição, uso de estratégias de aprendizagem e a autoavaliação do estudante (Mega et al., 2014).

As pesquisas de Pelissoni (2016) e de Rosário et al. (2016) aplicaram um programa de intervenção fundamentado na fase cíclica da autorregulação da aprendizagem Planejar, Executar e Avaliar (PLEA), que aborda os aspectos cognitivos, metacognitivos e motivacionais envolvidos nesse processo (Rosário, Núñez, \& González-Pienda, 2012). Este programa centralizou suas atividades nas reflexões promovidas pelas Cartas de Gervásio (Rosário et al., 2012), que propõem, dentre as suas diversas atividades, o estímulo às estratégias de aprendizagem e à autonomia do estudante. Ao final do programa, Pelissoni (2016) verificou algumas mudanças na percepção da vida acadêmica dos estudantes, em especial, na sua motivação, estratégias adotadas, no gerenciamento do tempo, comportamento, ambiente físico e social, afeto e estados emocionais. 
Rosário et al. (2016), avaliam a intervenção na ARA como eficaz, por proporcionar aos estudantes maior conhecimento e noções de uso das estratégias de autorregulação, por meio do estímulo às estratégias metacognitivas (e.g., habilidade de antecipar as consequências das ações em curto, médio e longo prazo), os recursos de gestão (e.g., organização do tempo, fazer anotações durante as aulas, evitar distrações, dentre outros) e a motivação (e.g., estabelecimento de metas e objetivos).

Mediante as acepções teóricas e empíricas dos construtos aqui abordados, o presente estudo pretendeu verificar as associações existentes entre as metas de realização e a autorregulação da aprendizagem, em nível de correlação e de predição em estudantes ingressantes. Adicionalmente, foram analisadas as possíveis diferenças entre ambos os construtos em razão da autopercepção de desempenho acadêmico desses estudantes. Partiu-se do pressuposto de que os estudantes orientados pela meta aprender e meta performance aproximação possivelmente seriam autorreguladores da sua aprendizagem, e que também apresentariam maior autopercepção de desempenho acadêmico, enquanto que aqueles guiados pela meta performance evitação demonstrariam maiores dificuldades em se autorregularem e se autoavaliariam de modo negativo. Enfatiza-se, ainda, principalmente o potencial preditivo da meta aprender para a autorregulação da aprendizagem.

\section{Método}

\section{Participantes}

A amostra do presente estudo contou com 404 estudantes universitários ingressantes de uma instituição de Ensino Superior localizada em um município do interior do Rio Grande do Sul. A média de idade dos participantes foi de 19,62 anos $(D P=4,870)$, sendo 235 mulheres e 170 homens. A maioria dos participantes eram estudantes do curso de Direito $(n=111)$, Psicologia $(n=73)$, Arquitetura e Urbanismo $(n=49)$, Engenharia Civil $(n=42)$, Medicina $(n=41)$. Em menor número havia os estudantes dos cursos de Odontologia, Ciência da Computação, Administração, Sistemas de Informação e Engenharia Mecânica, que juntos totalizaram 21,8\% da amostra. Quando questionados se o curso declarado era a primeira opção de escolha, 368 estudantes disseram que sim e apenas 32 que não. Portanto, a amostra foi composta majoritariamente por estudantes que estavam na universidade pela primeira vez $(n=349)$, sendo poucos os que iniciaram outro curso e abandonaram $(n=40)$, que estavam em uma segunda graduação $(n=8)$ e que estavam cursando duas graduações ao mesmo tempo $(n=3)$. A maioria dos estudantes frequentava a universidade no período noturno $(n=236)$, seguido do período diurno $(n=126)$ e uma pequena parcela em tempo integral $(n=41)$. A maior parte destes alunos $(n=243)$ declarou não exercer nenhum trabalho remunerado, ao passo que 156 disseram que o exerciam.

\section{Instrumentos}

Escala de Avaliação da Motivação para a Aprendizagem em Alunos Universitários (EMAPRE-U; Zenorini \& Santos, 2010): este instrumento afere a motivação para aprender de estudantes do Ensino Superior na perspectiva das metas de realização. A escala é composta por 28 itens divididos em três dimensões, sendo que cada uma delas representa uma meta de realização, a saber, meta aprender (12 itens), meta performance aproximação (9 itens) e meta performance evitação (7 itens). A EMAPRE-U é respondida por meio de uma chave de resposta do tipo Likert de 3 pontos, sendo 1, "concordo", 2 , "não sei" e 3 , "discordo". No que diz respeito às propriedades psicométricas desta escala, o estudo de Santos, Alcará e Zenorini (2013) verificou evidências de validade de critério e consistência interna, bem como estimativas de fidedignidade, com o coeficiente alfa variando entre 0,72 a 0,83. Já no estudo de Santos e Mognon (2016) o coeficiente alfa encontrado em uma amostra de universitários variou entre 0,86 a 0,91 . Para o presente estudo o coeficiente alfa demonstrou uma consistência interna satisfatória, sendo para a meta aprender, $\alpha=0,81$, meta performance aproximação, $\alpha=0,85$ e meta performance evitação, $\alpha=0,87$.

Inventário de Processos de Autorregulação da Aprendizagem (IPAA, Rosário et al., 2009): Este inventário avalia a autorregulação da aprendizagem com base no modelo cíclico proposto por Zimmerman (2000). O instrumento é unidimensional, composto por nove itens que avaliam as fases de planejamento, execução e de autoavaliação. A chave de resposta é em formato Likert, que varia de 1 "nunca" a 5 "sempre". Desse modo, altas pontuações no IPAA indicam que o estudante emprega a autorregulação da aprendizagem na rotina acadêmica e vice-versa. A aplicação pode ser feita de forma coletiva ou individual, sem tempo mínimo e máximo preestabelecido para o sujeito respondê-lo. Quanto às propriedades psicométricas do instrumento, o estudo de Rosário et al. (2009) apresentou coeficiente de consistência interna de $\alpha=0,87$ e de Paiva e Lourenço (2012) de $\alpha=0,82$, ambos com amostras de estudantes portugueses. A versão brasileira do instrumento, adaptada por Polydoro, Rosário, Sampaio e Freitas (2011), apresentam $\alpha=0,75$. 
Para o presente estudo o IPAA apresentou um coeficiente alfa satisfatório, com $\alpha=0,86$, obtida pela amostra de estudantes avaliada.

Ademais, foi solicitado aos alunos informação sobre como percebiam em média seu próprio rendimento no curso, em termos percentuais de aproveitamento, expressos pelas notas ou conceitos obtidos. Mediante esta orientação, os estudantes responderam classificando o seu próprio rendimento acadêmico a partir das seguintes classificações: entre inferior a 50 e até $59 \%$, de 60 a $79 \%$ e de 80 até $100 \%$.

\section{Procedimentos}

\section{Coleta de dados}

Após aprovação do projeto da presente pesquisa pelo Comitê de Ética em Pesquisa da Universidade o qual está vinculado (CAAE: 53189216.4.0000.5514), os estudantes que se dispuseram a participar espontaneamente do estudo assinaram o Termo de Consentimento Livre e Esclarecido (TCLE). A coleta de dados foi realizada seguindo a Resolução 466/12 que regulamenta a pesquisa em seres humanos (Brasil, 2016) e ocorreu mediante a anuência do diretor da Instituição de Ensino participante da pesquisa, bem como do coordenador de cada curso de graduação e dos professores responsáveis pelas aulas nos dias programados para a aplicação dos instrumentos. O questionário sociodemográfico, a EMAPRE-U e o IPAA foram aplicados em um tempo médio de 40 minutos, de forma coletiva e em horário normal de aula.

\section{Análise dos dados}

Os dados foram analisados no software Statistical Package for the Social Sciences (SPSS), versão 2.2. As análises descritivas foram utilizadas em vias de caracterizar a amostra e obter as médias e desvios padrão dos fatores da EMAPRE-U e do IPAA, com base nas pontuações da chave de resposta de ambos os instrumentos. Para correlacionar cada um dos fatores da EMAPRE-U e o IPAA, utilizou-se a prova de correlação $r$ de Pearson. Os índices de magnitude foram interpretados de acordo com Dancey e Ready (2013). A análise de regressão método enter foi empregada para verificar o potencial preditivo dos fatores da EMAPRE-U para o IPAA. Empregou-se a análise de quartis para dividir a avaliação de autopercepção de desempenho acadêmico dos estudantes em grupos de baixa, média e alta autopercepção. Também se utilizou a análise de variância ANOVA e a prova post hoc de Tukey com o intuito de averiguar as possíveis diferenças entre a EMAPRE-U e o IPAA em razão dos três grupos de autopercepção de desempenho acadêmico. Para examinar a consistência interna dos instrumentos aplicou-se a análise de confiabilidade alfa.

\section{Resultados}

Com a finalidade de atingir os objetivos propostos pelo presente estudo, inicialmente verificaram-se as médias e desvios padrão dos fatores da EMAPRE-U e do IPAA. Por meio da análise descritiva obtiveram-se os seguintes índices: EMAPRE-U - meta aprender, $M=$ $1,48(D P=0,377)$; meta performance aproximação, $M=$ $1,87(D P=0,550)$; meta performance evitação, $M=1,49$ $(D P=0,565)$; IPAA, $M=3,86(D P=0,756)$. Pelas análises descritivas, pode-se dizer que os estudantes possuíam um perfil motivacional orientado pela meta performance aproximação (média acima de 1,5), marcado pela preocupação do estudante em ser reconhecido pelos professores e colegas de turma por seus bons resultados, e utilizavam a autorregulação da aprendizagem de modo satisfatório (média acima de 2,5). Em seguida, correlacionou-se cada um dos fatores da EMAPRE-U com o IPAA. Os resultados são expostos na Tabela 1.

Tabela 1

Prova de correlação $r$ de Pearson entre a EMAPRE-U e o IPAA $(N=405)$

\begin{tabular}{ccccc}
\hline & & Meta aprender & $\begin{array}{c}\text { Meta performance } \\
\text { aproximação }\end{array}$ & $\begin{array}{c}\text { Meta performance } \\
\text { evitação }\end{array}$ \\
\hline \multirow{2}{*}{ Autorregulação da aprendizagem } & $r$ & 0,24 & 0,13 & 0,01 \\
& $p$ & 0,01 & 0,07 & 0,83 \\
\hline
\end{tabular}

Ao aplicar a prova de correlação $r$ de Pearson identificou correlação significativa, positiva e de fraca magnitude apenas entre a meta aprender e o IPAA $(r=0,24 ; p<$
0,01). Este índice, apesar de ser estatisticamente baixo, sugere que a meta aprender se associou às três fases da ARA. Ao se prepararem para as atividades, o estabelecimento de 
metas e o gerenciamento do tempo estão ligados ao nível de dedicação do estudante aos estudos, principalmente quando este confere um significado ao conteúdo que está sendo aprendido. Outros aspectos aqui identificados que favorecem o envolvimento do estudante com as atividades acadêmicas, foram a sua persistência diante de tarefas difíceis, bem como a possibilidade de, a partir da devolutiva feita pelo professor, ter mais noção sobre os pontos que podem ser melhorados.

Para examinar o quanto as metas de realização predizem a autorregulação da aprendizagem aplicou-se a análise de regressão método enter entre os fatores da EMAPRE-U (variável explicativa) e o IPAA (variável dependente). O resultado desta análise é apresentado na Tabela 2.

Tabela 2

Análise de regressão método enter para a EMAPRE-U e a IPAA

\begin{tabular}{clccc}
\hline Variável dependente & \multicolumn{1}{c}{ Variável explicativa } & $B$ & $p$ & $R^{2}$ ajustado \\
\hline \multirow{3}{*}{ IPAA } & Meta aprender & 0,344 & 0,01 & \\
& Meta performance aproximação & 0,140 & 0,04 & 0,07 \\
& Meta performance evitação & 0,014 & 0,16 & \\
\hline
\end{tabular}

Conforme a Tabela 2, as dimensões meta aprender e meta performance aproximação da EMAPRE-U representaram 7\% do nível explicativo do IPAA $(p<0,01)$. Por meio da Análise de Variância ANOVA se obteve o valor $F=9,870(3,401 ; p<0,01)$, o que indica que as essas duas metas de realização predizem a da autorregulação da aprendizagem. Verificou-se, ainda, por meio da correlação residual fornecida pelo teste de DurbinWatson, não haver correlação entre os resíduos, pois o valor identificado pelo teste foi de 1,872 , situando-se entre 1,5 e 2 , o que confere confiabilidade ao modelo (Field, 2009).

Esses índices de regressão sugerem que as características da meta aprender, relativas ao interesse em aprender coisas novas, ao ajustamento do esforço para a realização das tarefas de modo intencional e à persistência, são promotores da ARA, especialmente na definição dos objetivos que levam o aluno a estudar. Aspectos da meta performance aproximação, como estudar para se sair melhor que os colegas de turma ou liderar os grupos de trabalho, também preveem a ARA. Supõe-se que, apesar de o significado dado à tarefa pelo estudante orientado por essa meta estar muito mais atrelado em se sobressair na sala do que na qualidade da aprendizagem em si, a preocupação em ser um bom aluno favoreça alguns aspectos da ARA, principalmente na atenção dada as devolutivas das atividades avaliativas e na comparação de suas notas com as dos colegas de sala. Assim, a razão para se empenhar nos estudos, neste caso, pode estar na necessidade de se destacar em relação à turma.

Com base na análise dos quartis dos dados referentes à autopercepção de desempenho acadêmico dos estudantes foram criados três grupos. O Grupo 1 (G1) englobou os estudantes que consideraram que o seu desempenho acadêmico correspondia a um aproveitamento inferior a 50 e até $59 \%(n=43)$, o Grupo $2(\mathrm{G} 2)$, de 60 a $79 \%(n$ $=163)$ e o Grupo $3(\mathrm{G} 3)$, com $80 \%$ ou mais $(n=193)$. A Análise de Variância ANOVA foi aplicada com o intuito de examinar as diferenças entre os três grupos na EMAPRE-U e no IPAA, seguida da prova post hoc de Tukey. Os resultados estatisticamente significativos destas análises são apresentados na Tabela 3.

Quanto às metas de realização, os estudantes com autopercepção de desempenho acadêmico alta detêm mais características da meta aprender do que aqueles que apresentaram autopercepção de desempenho baixa, indicativo de características como a persistência, a satisfação por aprender coisas novas e de se sentir desafiado. Por sua vez, os estudantes com autopercepção de desempenho acadêmico baixa mostraram-se mais orientados pela meta performance evitação. Possivelmente, a autoavaliação negativa dos estudantes do G1 se deva por especificidades dessa meta, marcada pela sensação de inferioridade, receio de ser taxado como menos inteligente por professores e colegas, fazendo com que evitem participar ativamente das aulas e tirar dúvidas dos conteúdos trabalhados. Os resultados da meta performance aproximação não configuraram diferenças estatisticamente significativas, por apresentar valor $F=8,128(2,398 ; p>0,05)$. A prova post hoc de Tukey também não diferenciou os grupos de desempenho acadêmicos em subconjuntos para esta meta performance.

No que diz respeito ao IPAA, foi possível identificar a partir das médias expostas na Tabela 3, que os estudantes com autopercepção de desempenho acadêmico média e alta se apresentaram com maior potencial para autorregularem a sua aprendizagem do que aqueles classificados com baixa autopercepção de desempenho. Estes resultados 
sugerem que os estudantes do G2 e G3 quando comparados com o G1, possuem uma percepção de competência mais positiva para a aprendizagem e para obtenção do êxito acadêmico. Além disso, estão mais aptos a fazer uma análise crítica acerca dos objetivos traçados em cada disciplina e a identificar os pontos que podem ser melhorados.

Tabela 3

Análise de Variância ANOVA e prova post hoc de Tukey para o IPAA e a EMAPRE considerando a autopercepção de desempenho acadêmico

\begin{tabular}{|c|c|c|c|c|c|}
\hline \multirow{2}{*}{ Instrumentos } & \multirow{2}{*}{$F^{*}$} & \multirow{2}{*}{ Grupos } & \multicolumn{3}{|c|}{ Subgrupos } \\
\hline & & & $\mathrm{N}$ & 1 & 2 \\
\hline \multirow{3}{*}{ Meta aprender } & \multirow{3}{*}{$\begin{array}{c}9,223 \\
p<0,01\end{array}$} & G1 & 43 & 2,46 & \\
\hline & & $\mathrm{G} 2$ & 163 & 2,57 & 2,57 \\
\hline & & G3 & 193 & & 2,69 \\
\hline$P$ & & & & 0,12 & 0,08 \\
\hline \multirow{3}{*}{$\begin{array}{c}\text { Meta performance } \\
\text { evitação }\end{array}$} & \multirow{3}{*}{$\begin{array}{c}8,128 \\
p<0,01\end{array}$} & G1 & 43 & 1,66 & \\
\hline & & G2 & 163 & & 1,58 \\
\hline & & G3 & 193 & & 1,38 \\
\hline$P$ & & & & 1 & 0,62 \\
\hline \multirow{3}{*}{ IPAA } & \multirow{3}{*}{$\begin{array}{c}3,142 \\
p=0,04\end{array}$} & G1 & 43 & 3,58 & \\
\hline & & G2 & 163 & & 3,89 \\
\hline & & G3 & 193 & & 3,88 \\
\hline$P$ & & & & 1 & 0,99 \\
\hline
\end{tabular}

* Nota: Para todas as comparações, $g l=2$

\section{Discussão}

A respeito da motivação para aprender, os resultados da análise descritiva indicaram a prevalência da orientação pela meta performance aproximação, indicativo de que grande parte dos estudantes ingressantes avaliados percebia o seu próprio sucesso acadêmico pela comparação feita com os colegas de sala e pelo reconhecimento dos professores. Em razão disso, possivelmente valorizam o fato de tirarem notas mais altas e de assumirem a liderança dos grupos de estudos que eventualmente participem (Bzuneck et al., 2014; Bzuneck \& Boruchovitch, 2016; Pekrun, 2016). Estes dados também sugerem que, no geral, a amostra avaliada emprega os componentes que constituem a autorregulação da aprendizagem (ARA) nos níveis de planejamento, execução e avaliação, avaliada pelo IPAA, na realização das atividades acadêmicas (Rosário et al., 2016; Zimmerman et al., 2015).

A associação entre a meta aprender e as fases da ARA identificadas neste estudo, pode estar ligada às características apresentadas por esse perfil motivacional, em especial ao investimento do esforço e persistência nas atividades acadêmicas, o que se reflete na organização do aluno para o estudo, na utilização de estratégias de aprendizagem mais eficazes e na capacidade de se autoavaliar (Mega et al., 2014; Rosas, 2015). Ao enfatizar este último aspecto, pondera-se que a orientação pela meta aprender pode possibilitar que o estudante seja capaz de desenvolver habilidades que corroborem a sua aprendizagem, conseguindo mais controle das situações e maior nível de intencionalidade (Bzuneck et al. 2014; Weiner, 2010).

A ausência de correlações estatisticamente significativas entre as metas performances e a ARA se justifica pela oposição dos comportamentos apresentados pelo estudante guiado por essas metas e as características que definem o aluno autorregulador da sua aprendizagem. Um dos aspectos que definem a ARA é a contínua autoavaliação feita pelo estudante acerca daquilo que pode ser melhorado e pela sua crença em sua capacidade de aprender (Bzuneck et al., 2014; Mega et al., 2014).

Estes aspectos diferem das crenças que sustentam as metas performances, especialmente na meta performance evitação, na qual a preocupação motivacional decorre da insegurança de ser mal avaliado, o que repercute negativamente na sua percepção de competência, e na autoavalição negativa feita pelo estudante (Bzuneck et al., 2014; Rosas, 
2015). Por outro lado, verificou-se que a meta performance aproximação somada à meta aprender predizem a ARA. Ao focalizar a meta performance aproximação, é importante destacar que a preocupação em tirar boas notas para se sentir valorizado faz com que o estudante se esforce e adquira estratégias de aprendizagem que corroboram o bom desempenho acadêmico (Bzuneck, 2009).

Sobre a contribuição preditiva das metas aprender e performance aproximação para a ARA, é importante ponderar sobre dois pontos referentes ao pequeno nível explicativo obtido ( $7 \%$ da variância explicativa). O primeiro deles refere-se ao aspecto teórico do ciclo da autorregulação da aprendizagem proposto por Zimmerman (2000, 2013). Como mencionado na introdução, este modelo agrega diversos construtos de cunho motivacional e outros, como a cognição e a metacognição (Shunk et al., 2013; Zimmerman, 2011). Neste sentido, é compreensível que a contribuição preditiva das metas de realização para a ARA seja quantitativamente menor por estar associada a outros construtos da motivação para aprender, como é o caso da autoeficácia e das atribuições de causalidade (Zimmerman et al., 2015). Portanto, conjectura-se que o potencial preditivo da motivação para aprender para a ARA poderia aumentar com o acréscimo dos demais construtos motivacionais, que teoricamente a constituem.

$\mathrm{O}$ segundo aspecto diz respeito a uma característica do instrumento utilizado para avaliar a autorregulação da aprendizagem. Dos nove itens que compõem o IPAA, grande parte deles não aborda a motivação para aprender de forma direta. Seus itens a focalizam por meio dos componentes cognitivo, metacognitivo, autoeficácia e comportamental (a ação em si), o que poderia ser a razão do baixo percentual de predição encontrado. Ainda assim, é importante salientar que o IPAA apresentou consistência interna satisfatória para a amostra avaliada, o que confere, em termos psicométricos, estimativa de fidedignidade ao instrumento.

No que tange às limitações deste estudo, destaca-se o fato de não ter havido acesso às notas obtidas pelos estudantes ou ao seu coeficiente de rendimento acadêmico, o que forneceria um parâmetro mais realista de seu desempenho acadêmico (Daniels et al., 2013; Mega et al., 2014; Rosas, 2015; Wolters \& Hussain, 2014). Aqui foi considerada apenas a percepção do estudante sobre o seu próprio desempenho acadêmico que, apesar de ser uma variável importante do autojulgamento do estudante acerca das suas competências acadêmicas, conforme assinalado por Weiner (2010), tal informação pode não ser suficiente para aferir o seu real desempenho.

Outra limitação diz respeito ao fato de que a composição da amostra de universitários contou apenas com respondentes de uma única instituição privada. Sugere-se que em futuras pesquisas sejam avaliados alunos de outras localidades do Brasil e de instituições públicas, bem como aqueles que estejam cursando o Ensino Superior à distância, nas modalidades parcial ou integral. Também seria interessante investigar estudantes que já se encontram há algum tempo na universidade, a fim de verificar a possível existência de diferenças nas metas de realização e na ARA de alunos ingressantes com os que estão no meio do curso, os concluintes e, também aqueles que foram reprovados (dependências).

Além disso, será importante ampliar o conhecimento sobre os aspectos relacionados ao modo como os estudantes pensam, se organizam e agem em relação às atividades e ao espaço acadêmico. A extensão das investigações à variáveis de cunho afetivo-emocional, certamente possibilitarão avaliar como elas se associam às crenças motivacionais e à ARA. Também vale incluir na agenda de pesquisa, a possibilidade de focalizar outros elementos relacionados ao processo de ensino e aprendizagem, que podem ser de cunho motivacional, como as atribuições de causalidade, e a adaptação acadêmica, sobretudo ao avaliar estudantes ingressantes. Sugere-se, ainda, a avaliação de construtos relacionados ao campo da Orientação Profissional e de Carreira, como a adaptabilidade e a autoeficácia para decisão de carreira, o que permitiria uma interlocução entre esta área e a Psicologia Escolar Educacional, em termos de conhecimento e possibilidades de elaboração e aplicação de intervenções em benefício do estudante.

Em termos de aplicação prática, verifica-se que com o uso de instrumentos de avaliação, tal como os utilizados neste estudo, é possível delimitar os perfis motivacionais dos estudantes e conhecer as características de sua autorregulação da aprendizagem. Sabendo-se que ambos podem ser alterados, abre-se amplo espaço de atuação para os próprios professores e para os serviços de orientação da IES, que poderão oferecer alternativas para que essa mudança ocorra, tornando os alunos mais preparados para atuar no mundo do trabalho, com a interlocução entre teoria e prática e o estímulo à curiosidade.

\section{Referências}

Anderman, E. M., Anderman, L. H., Yough, M. S., \& Gimbert, B. G. (2010). Value-added models of assessment: Implications for motivation and accountability. Educational Psychologist, 45(2), 123-137. doi:10.1080/00461521003703045 
Brasil, Conselho Nacional de Saúde. (2016). Resolução CNS 510, de 07 de abril de 2016. Recuperado de http://conselho. saude.gov.br/resolucoes/2016/Reso510.pdf

Bzuneck, J. A. (2009). A motivação do aluno orientado a metas de realização. Em E. Boruchovitch, \& J. A. Bzuneck (Orgs.). A motivação do aluno: contribuições da Psicologia contemporânea (pp. 58-77). Petrópolis, RJ: Vozes.

Bzuneck, J. A., \& Boruchovitch, E. (2016). Motivação e autorregulação da motivação no contexto educativo. Psicologia Ensino \& Formação, 7(2), 73-84. doi: 10.21826/2179-58002016727584

Bzuneck, J. A., Boruchovitch, E., Miranda, L., \& Almeida, L. S. (2014). Motivação acadêmica dos alunos. In L. S. Almeida, \& A. M. Araújo (Eds.). Aprendizagem e sucesso escolar: Variáveis pessoais dos alunos (pp. 173-214). Braga: ADIPSIEDUC.

Dancey, C. P., \& Reidy, J. (2013). Estatística sem matemática para psicólogos. São Paulo: Penso Editora.

Daniels, L. M., Haynes, T. L., Stupnisky, R. H., Perry, R. P., Newall, N., \& Pekrun, R. (2008). Individual differences in achievement goals: A longitudinal study of cognitive, emotional, and achievement outcomes. Contemporary Educational Psychology, 33, 584-608. doi: 10.1016/j.cedpsych.2007.08.002.

Daniels, L. M., Perry, R. P., Stupnisky, R. H., Stewart, T. L., Newall, N. E., \& Clifton, R. A. (2013). The longitudinal effects of achievement goals and perceived control on university student achievement. European Journal of Psychology of Education, 29(2), 175-194. doi: 10.1007/s10212-013-0193-2

Ferradás, M. M., Freire, C., Núñez, J. C., Piñeiro, I., \& Rosário, P. (2017). Motivational profiles in university students. Its relationship with self-handicapping and defensive pessimism strategies. Learning and Individual Differences, 56, 128-135. doi: 10.1016/j.lindif.2016.10.018

Field, A. (2009). Descobrindo a estatística usando o SPSS. Porto Alegre: Artmed.

Grant, H., \& Dweck, C. S. (2003). Clarifying achievement goals and their impact. Journal of Personality and Social Psychology, 85(3), 541-553. doi: 10.1037/0022-3514.85.3.541

Mega, C., Ronconi, L., \& De Beni, R. (2014). What makes a good student? How emotions, self-regulated learning, and motivation contribute to academic achievement. Journal of Educational Psychology, 106(1), 121-131. doi: 10.1037/a0033546

Paiva, M. O. A., \& Lourenço, A. A. (2012). A influência da aprendizagem autorregulada na mestria escolar. Estudos e Pesquisas em Psicologia, 12(2), 501-520. Recuperado de http://www.revispsi.uerj.br/v12n2/artigos/pdf/ v12n2a11.pdf

Pelissoni, A. M. S. (2016). Eficácia de um programa híbrido de promoção da autorregulação da aprendizagem para estudantes do ensino superior. (Tese de Doutorado). Unicamp: Campinas, SP.

Pekrun, R. (2016). Academic emotions. In K. R. Wentzel \& D. B. Miele (Eds.), Handbook of Motivation at School (pp. 120-144). New York and London: Routledge.

Polydoro, S. A. J., Rosário, P., Sampaio, R. K. N., \& Freitas, F. A. (2011). Sucesso no ensino superior e variáveis envolvidas. Anais do X Congresso Nacional de Psicologia Escolar e Educacional (CONPE): Caminhos trilhados, caminhos a percorrer, Maringá, PR, 40.

Rosário, P., Fuentes, S., Beuchat, M., \& Ramaciotti, A. (2016). Self-regulated learning in a college classroom: A curriculum infusion approach. Revista de Investigación Educativa, 34(1), 31-49. doi: 10.6018/rie.34.1.229421

Rosário, P., Lourenço, A. A., Paiva, M. O., Núñez, J. C., González-Pienda, J., \& Valle, A. (2009). Inventário de processos de autorregulação da aprendizagem (IPAA). In C. Machado, M. M. Gonçalves, L. S. Almeida, \& M. R. Simões (Orgs.), Instrumentos e Contextos da Avaliação Psicológica (pp. 159-174). Coimbra, Portugal: Almedina.

Rosário, P. Núñez, J. C., \& González-Pienda, J. (2012). Cartas do Gervásio ao seu Umbigo: comprometer-se com o Estudar na Educação Superior. São Paulo: Almedina Editores.

Rosas, J. S. (2015). Validation of the achievement goal questionnaire-revised in argentinean university students (A-AGQ-R). International Journal of Psychological Research, 8(1), 10-23. Recuperado de http://www.scielo.org. co/pdf/ijpr/v8n1/v8n1a02.pdf

Santos,A.A.A.,Alcará,A.R., \&Zenorini, R. D.P.C.(2013). Estudos psicométricos da escala de motivação para a aprendizagem de universitários. Fractal: Revista de Psicologia, 25(3), 531-546. doi: 10.1590/S1984-02922013000300008

Santos, A. A. A., \& Mognon, J. F. (2016). Motivation assessment scale for learning in higher education: Validity evidence. Psico-USF, 21(1), 101-110. doi:10.1590/1413-82712016210109.

Schunk, D. H., Usher, E. L., \& Barry, J. (2013). Zimmerman's theory of self-regulated learning. In H. Bembenutty, T. J. Cleary, \& A. Kitsantas (Eds.), Applications of self-regulated learning across diverse disciplines: A tribute to Barry J. Zimmerman (pp. 1-28). Charlotte, NC: Information Age Publishing. 
Zenorini, R. P. C., \& Santos, A. A. A. (2010). Escala de Metas de Realização como medida da motivação para a aprendizagem. Interamerican Journal of Psychology, 44(2), 291-298. Recuperado de http://www.redalyc.org/ pdf/284/28420641010.pdf

Zimmerman, B. J. (2000). Attaining self-regulation: A social cognitive perspective. Em M. Boekaerts, P. R. Pintrich, \& M. Zeidner (Eds.), Handbook of selfregulation (pp. 13-39). San Diego: Academic Press.

Zimmerman, B. J. (2011). Motivational sources and outcomes of self-regulated learning and performance. In B. J. Zimmerman, \& D. H. Schunk (Eds.), Handbook of self-regulation of learning and performance (pp. 49-64). New York: Routledge.

Zimmerman, B. J. (2013). From cognitive modeling to self-regulation: A social cognitive career path. Educational Psychologist, 48(3), 135-147. doi: 10.1080/00461520.2013.794676

Zimmerman, B. J, Schunk, D. H., \& DiBenedetto, M. K. A. (2015). A Personal agency view of self-regulated learning. Self-Concept, Motivation and Identity: Underpinning Success with Research and Practice. In F Guay, H. Marsh, D. M. Mclnerney, \& R. G. Craven (Eds.), Self-Concept, Motivation, and Identity: Underpinning Success with Research and Practice (pp. 83-114). Charlotte, NC: Information Age Publishing.

Weiner, B. (2010). The development of an attribution-based theory of motivation: A history of ideas. Educational Psychologist, 45(1), 28-36. doi: 10.1080/00461520903433596

Wolters, C. A., \& Hussain, M. (2015). Investigating grit and its relations with college students' self-regulated learning and academic achievement. Metacognition and Learning, 10(3), 293-311. doi: 10.1007/s11409-014-9128-9

Recebido 30/01/2018

Reformulado 03/06/2018

Aceite Final 07/10/2018

Sobre as autoras

Simone Nenê Portela Dalbosco é psicóloga e Doutora em Psicologia pela Universidade São Francisco, Campus de Campinas/SP.

Adriana Satico Ferraz é psicóloga e mestranda em Psicologia pela Universidade São Francisco, Campus de Campinas/SP, com bolsa da Fundação de Amparo de Pesquisa do Estado de São Paulo (FAPESP).

Acácia Aparecida Angeli dos Santos é psicóloga e Doutora em Psicologia Escolar e do Desenvolvimento Humano pela USP. Atualmente é docente do programa de Pós-Graduação Stricto Sensu em Psicologia da USF, Campus de Campinas/SP e Bolsista produtividade do CNPq 1A. 